\title{
Energy Drinks May Improve Postural Sway While Provoking an Early Onset of Muscle Fatigue
}

Ashley Correa SPT, Clare Hanrahan SPT, Lauren Basye SPT \& Martín G. Rosario* PT, PhD, CSFI, ATRIC

School of Physical Therapy, Texas Woman's University, Dallas Campus; 5500 Southwestern Medical Ave. Dallas, United States.

Article Details
Article Type: Research Article
Received date: $16^{\text {th }}$ January, 2021
Accepted date: $29^{\text {th }}$ March, 2021
Published date: $19^{\text {th }}$ April, 2021
Corresponding Author: Martín G. Rosario PT, PhD, CSFI, ATRIC, Assistant Professor, School of Physical Therapy,
Texas Woman's University, Dallas Campus; 5500 Southwestern Medical Ave. Dallas, TX 75235-72. United States.
E-mail: mrosario1@twu.edu
Citation: Correa, A., Hanrahan, C, Basye, L., \& Rosario, M.G. (2021). Energy Drinks May Improve Postural Sway While
Provoking an Early Onset of Muscle Fatigue. J Pub Health Issue Pract 5(1): 176. doi: https://doi.org/10.33790/jphip1100176
Copyright: $\odot 2021$, This is an open-access article distributed under the terms of the Creative Commons Attribution License
4.0, which permits unrestricted use, distribution, and reproduction in any medium, provided the original author and source
are credited.

\section{Abstract}

Background: In recent years, energy drink consumption (EDC) has flared up among college students in the 18-34-year-old male demographic. Energy drinks cause balance alterations, reduction of blood flow, and distort lower limb neuromuscular activation.

Purpose/Objective: We strived to discover which specific additive among three different drinks (Red Bull, Rockstar, Bang) could contribute to abnormalities in stability, memory, and muscle activation. Therefore, this investigation's aim is two-fold. First, we assessed the impact of EDC on muscle activation/fatigue and standing balance during multitasking activities. Second, we propose to determine the effect of EDC on memory during multitasking activities.

Methods: Twenty healthy young adults ranging from 22 to 28 years old, took part in the study. Demographic and cardiovascular data were collected, and later all participants completed cognitive evaluations (memory, motor speed) before the EDC. We obtained neuromuscular data via EMG sensors placed on hand grip muscles (wrist flexors) before performing the balance (no foam/foam, EO/EC) and motor (while holding a water cup; no nodding/nodding) tasks measured by accelerometers. Each participant completed all tasks pre- and post16-ounce EDC. A MANOVA analysis was implemented to compare pre- and post-data. Statistical significance was set at $p<0.05$.

Results: Among the three diverse drinks, 1) A trend to reduce jerk movements (postural sway) was detected mostly in the anteriorposterior (AP) direction with the Red Bull group and 2) Handgrip neuromuscular data revealed an adaptation in diverse timing variables, more noticeable with the Red Bull group during muscle timing decay. 3) Finally, the memory protocol yielded no results after EDC.

Conclusions: While this study's outcomes did not show a substantial distinction between pre and post-EDC for the variables measured, among the three drinks, Red bull exhibited alterations. The reduction in EMG activity decay in the Red Bull group may suggest an early onset of muscle fatigue following EDC. Nevertheless, it seems that the reduced sway compensates for this early decay during the balance tests.

Clinical relevance: This study pointed out that some of the ingredients in Red Bull alter muscle activation and balance. Further research is warranted to gain a better understanding of the effects of Red Bull's ingredients on muscle fatigue and balance.

Key Words: energy drinks, muscle activation, memory, young adults

\section{Introduction}

Energy drinks (EDs) are beverages that are proclaimed to be capable of enhancing athletic performance, concentration, reaction time, and basal metabolic rate besides enhancing energy levels [1]. Energy drink consumption has gained in popularity, with sales rising by $240 \%$ between 2004 and 2009 [1]. Most consumers are in the demographic of young adult males between the ages of and 18-34 years old, with up to $57 \%$ of college students reporting ED use in one survey [1].

With energy drinks being extensively used in today's society as a mechanism for an instantaneous energy burst, it is essential that the benefits and dangers, as well as ingredients, are considered. However, because they are classified as a supplement, EDs are not regulated in the US [1]. The most widely studied ED ingredient is caffeine, shown to enhance alertness and mood and counteract the effects of sleep loss. On the other hand, caffeine can also cause gastrointestinal upset, nausea, insomnia, and withdrawal symptoms, including headaches. Caffeine toxicity is always a possibility of energy drink consumption, which is why the FDA has imposed a limit of $71 \mathrm{mg}$ of caffeine per 12 fluid ounces of soda [2]. However, energy drink manufacturers attempt to evade this regulation by marketing their products as "natural dietary supplements" [2].

Apart from caffeine, EDs typically have one or more of the following ingredients: taurine, guarana, glucuronolactone, ginseng, and L-carnitine. Most individuals are mindful of caffeine intake; however, many other ingredients are mostly unknown [3].

One article remarks the possible neuroprotective effects of taurine on aging brains [4]. There is limited evidence to confirm the cardiovascular safety of these ingredients [3,5]. Additionally, several of these additives have vasoactive properties, and the consequences of mixing these ingredients when ingested together have not been well studied [6]. Risks of energy drink consumption are a current issue due to the rise in ER visits compared to energy drink abuse. As stated in a recent article, "Through out 2007-2011, ED [energy drink] related emergency department visits in the United States doubled" [3]. Most of the reported effects of EDs are related to the cardiovascular system.

Numerous studies have documented a common thread of adverse cardiovascular responses seen with energy drink consumption. These events include abnormal heart rhythms like atrial fibrillation, ventricular fibrillation, ST elevation, and QT prolongation. 
Using energy drinks, such as Rock Star, has also been shown to increase blood pressure and the level of catecholamines (stress hormones released by the adrenal gland) in the blood, which are considered risk factors for cardiovascular events [7]. Other cardiovascular side effects have increased diastolic BP, increased incidence of heart palpitations, and increased platelet aggregation [8]. Additionally, many EDs contain carnitine, linked to atherosclerosis, and many of these drinks can trigger endothelial dysfunction [6].

The reasons behind the above mentioned are unclear, but the correlation between adverse effects and EDs is well documented. Caffeine exclusively does not indicate inducing any ECG changes; consequently, in-depth knowledge of the other primary ingredients in EDs is warranted.

Outside the cardiovascular system, EDs have been shown to bear effects on other body systems. It is common for EDs to contain high amounts of sugar, which may raise the risk of obesity, hypertension, and dental problems [9]., caffeine can lower the body's insulin resistance, placing consumers of EDs at higher risk for type II diabetes [9]. In the neurological system, EDs have been linked with an increase in risk for addiction and have been associated with increased impulsivity and reduced academic performance in college students [10].

Despite these adverse effects, there may be a slight benefit to EDs in terms of increasing muscle strength. One study found that ingestion of caffeinated EDs increased maximal voluntary isometric contraction strength in young, healthy males [11]. Another study identified that caffeine and ED intake improved sprinting performance and improved reaction time and agility in young, healthy males [12]. While some studies have shown a link between caffeine and increased repetition and power output during exercise [12], others have identified no change in these outcomes between EDs and placebo [13]. For instance, in a 2015 study, Trevino et al. [17] observed no distinction in biceps muscle activation after energy drink ingestion.

The most popular brands of energy drinks advertise [14] the benefits of "mental-focus" and "performance." Nevertheless, to the best of our knowledge, these are unproven claims. The principal ingredient in EDs is caffeine, which is the most used psychoactive drug in the world [10]. Caffeine, by itself can stimulate the central nervous system [15]. As mentioned, EDs often contain other ingredients such as taurine, guarana, and riboflavin, among others, which can alter the central nervous system in harmful matter [16, \&17] Merely a few studies have approached the connection between energy drinks and caffeine with the central nervous system (CNS) $[3,5,9,11,12,15$, 18]. Diminished blood flow [19] to peripheral structures, including muscles and nerves of the extremities, could potentially increase fatigue, thus altering the standing balance.

Considering all the above, during the first component of this investigation, we endeavor to determine the impact of EDs on balance and muscle activation/fatigue during multitasking activities. Standing balance [20] is under the influenced of the central nervous system (CNS) and motor control is achieved by the interaction of three principal systems within the CNS (visual, somatosensory, and vestibular). It is necessary to identify the association between caffeine and EDs on balance and lower extremity muscle activation. Because, reduced blood flow to the brain-cognitive areas could potentially induce alterations in memory. The second component of this study aimed to assess the association between caffeine and EDs on memory-attention while multitasking activities.

The question this study attempts to answer is: Do EDs alter memory, muscle activation, and balance? We hypothesize that EDs will potentially increase memory; nonetheless, reduce the period of focus/memory combined with a faster onset of muscle fatigue and a decrease in balance. This study will add to the gap related to the harmful effects of EDs on memory and muscle activity/fatigue that might conceivably affect students' learning and health.

\section{Methods}

We recruited 20 healthy adults, 2 males and 17 females. Age ranged from to 22-28 years old, with an average age of 23.9 years old $(+/-$ 1.85 years, Table 1 ) and all participants were TWU students currently enrolled in PT, OT or nursing programs. Exclusion criteria included a history of diabetes, heart conditions, and injuries or surgeries of the arm, elbow, or leg six months prior to the study.

\begin{tabular}{|l|l|l|l|}
\hline \multicolumn{5}{|l|}{ Characteristics of participants } \\
\hline Characteristics & Redbull $(\mathrm{n}=6)$ & Bang $(\mathrm{n}=7)$ & Rockstar $(\mathrm{n}=7)$ \\
\hline Age & $\mathrm{m}=24.4 \pm 1.2$ & $\mathrm{~m}=24.2+/-2.4$ & $\mathrm{~m}=23.7+/-2.0$ \\
\hline Male & $\mathrm{n}=4$ & $\mathrm{n}=0$ & $\mathrm{n}=1$ \\
\hline Female & $\mathrm{n}=2$ & $\mathrm{n}=7$ & $\mathrm{n}=6$ \\
\hline Height & $\mathrm{m}=66.1$ inches & $\mathrm{m}=66.8+/-2.5$ inches & $\mathrm{m}=66.7+/-3.1$ inches \\
\hline Weight & $\mathrm{m}=148.4$ pounds & $\mathrm{m}=140+/-13.7$ pounds & $\mathrm{m}=152.7+/-23.1$ pounds \\
\hline BMI & $\mathrm{m}=23.8 \pm 5.7$ & $\mathrm{~m}=22.1+/-$ & $\mathrm{m}=24.1+/-$ \\
\hline $\begin{array}{l}\text { Results of paired sample t-tests performed between pre-energy drink and post energy drink } \\
\text { cardiovascular data. Significance level set at } \mathrm{p} \leq 0.05 \text {. }\end{array}$ \\
\hline \multicolumn{4}{|r}{ Table 1: Characteristics of the study participants. } \\
\hline
\end{tabular}

After signing the informed consent and prior to drinking the energy drink, participants were asked to perform a memory protocol with four components: a) memory registration was assessed by repeating four items (for instance, house, blue, dog, sky), b) motor speed by a timed quick finger tapping over 5 seconds, c) psychomotor speed by a timed alternating hand sequence test over $10 \mathrm{~s}$ and $\mathrm{d}$ ) memory recall by remembering the four items on (a) after $2 \mathrm{~min}$.

Then, the participants were instrumented with EMG sensors to measure neuromuscular activity. Neuromuscular data, including muscle fatigue, were measured with a surface EMG sensor placed at the dominant medial epicondyle, at the elbow joint, and dominant side leg muscles (tibialis anterior and gastrocnemius). Next, to measure muscle activation/fatigue on wrist-hand muscles, participants were asked to hold a dynamometer for 10 seconds and rest for 10 seconds. This sequence was repeated three times for each participant.
Pressure platforms and accelerometers were used to record balance data, including changes in the distance and velocity of the center of pressure deviation during the balance protocol. For the balance protocol, participants were first instructed to stand for $15 \mathrm{~s}$ while holding a cup full of water in the dominant hand for the following conditions: a) stand on a firm surface with eyes open, while focusing on a marked spot on the wall at eye level, to set a baseline. Other balance tasks included b) standing on a foam (unstable) surface with eyes open; c) standing on foam (unstable) surface with eyes closed; d) standing on foam (unstable) surface with eyes open while nodding head up and down at $60 \mathrm{bpm}$ (gaze still fixated on mark on the wall), e) standing on a foam (unstable) surface nodding head up and down at $60 \mathrm{bpm}$ with eyes closed. During the nodding tasks, participants were instructed to find and maintain the rhythm before beginning the test. The EMG sensors recorded muscle activity/fatigue on the leg muscles (tibialis anterior and gastrocnemius). 
Next, participants drank the 16-ounce energy drink (either Rock Star, Red Bull, or Bang) at their own pace. After cardiovascular changes were detected, for instance, an increase in heart rate of 10 beats per minute, the memory protocol, grip strength, and balance protocol were repeated to compare pre-energy drink and post-energy drink data.

Data Analysis: Version 25 of the Statistical Package for the Social Sciences (SPSS) will be used for all data analysis. First, an ANOVA comparison was conducted with all the variables of interest between pre and post-energy drink consumption. I) EMG muscle activation wrist flexor data included 1) duration (sec), 2) time to a maximum peak of activation (sec), and 3) time to decay maximum activation (sec). II) Balance data included anterior-posterior and medio-lateral sway (inches) and sway. The velocities of the AP and ML sways (inches/meters) will also be variables of interest in this study. III) Hand grip strength in pounds of pressure. Finally, pre and post-time and performance data of the memory protocol were compared. $\mathrm{P}$ values $<.05$ will be accepted as statistically significant. Gathering this information will help determine the impact of the energy drink on balance, muscle activity and memory in young adults.

\section{Results}

Table 1 shows the demographic characteristics of the research participants, including age, gender, and BMI. Demographic data for the Red Bull, Bang, and Rockstar groups were comparable across all three groups.

Table 2 illustrates the results of the participant cardiovascular data. In the Redbull group, the average blood pressure, heart rate, and oxygen saturation increased after EDC. In the Bang group, the average blood pressure also increased after EDC; however, the average heart rate and oxygen saturation decreased after EDC. With Rockstar, the average blood pressure and heart rate increased after EDC, and oxygen saturation decreased slightly. In summary, average blood pressure increased across all three groups post EDC, while heart rate and oxygen saturation changes varied.

\begin{tabular}{|c|c|c|}
\hline \multicolumn{3}{|l|}{ Cardiovascular Data } \\
\hline Redbull & Pre Edrink & Post Edrink \\
\hline Systolic $(\mathrm{mmHg})$ & $112.8+-10.87$ & $117.6+/-18.64$ \\
\hline Diastolic (mmHg) & $74.4+/-6.46$ & $78.8+/-5.80$ \\
\hline Heart Rate (beats per minute) & $70.4+/-5.17$ & $80+/-8.80$ \\
\hline Oxygen Saturation (\%) & $97.6+/-1.51$ & $98+/-1.41$ \\
\hline Hand grip average & $66.69+/-19.16$ & $63.75+/-18.15$ \\
\hline Time for Cardio Change (minutes) & \multicolumn{2}{|l|}{$11.8+/-5.44$} \\
\hline Bang & Pre Edrink & Post Edrink \\
\hline Systolic $(\mathrm{mmHg})$ & $113.14+/-8.13$ & $114.85+/-7.42$ \\
\hline Diastolic (mmHg) & $78.14+/-6.44$ & $83.28+/-9.70$ \\
\hline Heart Rate (beats per minute) & $73+/-17.27$ & $70.28+/-11.27$ \\
\hline Oxygen Saturation (\%) & $98+/-1$ & $97.14+/-2.26$ \\
\hline Hand grip average & $58.31+/-9.1$ & $62.18+/-6.61$ \\
\hline Time for Cardio Change (minutes) & \multicolumn{2}{|l|}{$13.71+/-3.14$} \\
\hline Rockstar & Pre Edrink & Post Edrink \\
\hline Systolic (mmHg) & $117+/-11.81$ & $121.14+/-12.95$ \\
\hline Diastolic (mmHg) & $75.14+/-3.93$ & $87.14+/-6.03$ \\
\hline Heart Rate (beats per minute) & $73.71+/-15.15$ & $78.85+/-18.95$ \\
\hline Oxygen Saturation (\%) & $96.57+/-3.35$ & $96.42+/-2.37$ \\
\hline Hand grip average & $59.01+/-10.24$ & $60.63+/-9.24$ \\
\hline Time for Cardio Change (minutes) & \multicolumn{2}{|l|}{$13+/-2.44$} \\
\hline
\end{tabular}

Table 2: Characteristics of participants cardiovascular Data.

Tables 3-4 reveal the outcomes of postural sway during the different balance test for all participants, pre and post-energy drink. The results indicate that all values were comparable among the different drinks. However, although not significant, a tendency to reduce jerk movements was observed. This reduction was observed mostly in the AP jerk direction and with the Red Bull drink.

\begin{tabular}{|l|l|l|l|}
\hline AP Jerk $\left(\mathbf{m}^{2} / \mathbf{s}^{\mathbf{5}}\right)$ & Pre Edrink & Post Edrink & P value \\
\hline Redbull & $5.0463+/-10.0766$ & $1.7321+/-1.7136$ & 0.16 \\
\hline EO & $2.5232+/-1.5593$ & $1.7865+/-0.4774$ & 0.21 \\
\hline EC & $7.8435+/-10.5433$ & $3.6913+/-2.2392$ & 0.14 \\
\hline EOHUD & $10.2883+/-14.4565$ & $7.0148+/-6.2325$ & 0.44 \\
\hline ECHUD & Pre Edrink & Post Edrink & Pvalue \\
\hline Bang &
\end{tabular}

Table 3 to be cont... 


\begin{tabular}{|l|l|l|l|}
\hline EO & $1.0698+/-0.7981$ & $1.2800+/-1.9037$ & 0.92 \\
\hline EC & $2.1633+/-1.4410$ & $1.6214+/-1.0104$ & 0.32 \\
\hline EOHUD & $2.5047+/-1.6161$ & $2.0503+/-1.2800$ & 0.89 \\
\hline ECHUD & $2.9157+/-1.0776$ & $3.8996+/-2.3524$ & 0.80 \\
\hline Rockstar & Pre Edrink & Post Edrink & P value \\
\hline EO & $1.1952+/-0.5595$ & $0.9805+/-0.2583$ & 0.92 \\
\hline EC & $2.4239+/-0.5360$ & $1.7443+/-0.3799$ & 0.22 \\
\hline EOHUD & $5.3003+/-3.8876$ & $4.1020+/-3.2003$ & 0.64 \\
\hline ECHUD & $7.6217+/-7.0332$ & $6.7059+/-4.6263$ & 0.81 \\
\hline
\end{tabular}

$\mathrm{EO}=$ Eyes Open, $\mathrm{EC}=$ Eyes Closed, $\mathrm{HUD}=$ Head up and down, Motor $=$ holding a cup filled with water Table 3: Anterior-posterior Jerk during balance tasks. Results of repeated measure ANOVA between preenergy drink and post-energy drink. Significance level set at $\mathrm{p} \leq 0.05$.

\begin{tabular}{|c|c|c|c|}
\hline \multicolumn{4}{|c|}{ MLJerk $\left(\mathrm{m}^{2} / \mathbf{s}^{5}\right)$} \\
\hline Redbull & Pre Edrink & Post Edrink & Pvalue \\
\hline EO & $1.3612+/-1.6123$ & $0.7036+/-1.1814$ & 0.33 \\
\hline $\mathrm{EC}$ & $1.4817+/-1.4271$ & $1.6390+/-2.1405$ & 0.86 \\
\hline EOHUD & $1.3401+/-1.4813$ & $0.6690+/-0.4117$ & 0.17 \\
\hline ECHUD & $2.7283+/-2.9813$ & $1.7800+/-1.2001$ & 0.34 \\
\hline Bang & Pre Edrink & Post Edrink & Pvalue \\
\hline EO & $1.1171+/-0.7711$ & $1.0001+/-1.7705$ & 0.85 \\
\hline $\mathrm{EC}$ & $1.4389+/-0.9965$ & $1.6781+/-2.4662$ & 0.77 \\
\hline EOHUD & $0.9699+/-0.7972$ & $0.9293+/-0.6555$ & 0.93 \\
\hline ECHUD & $1.2950+/-0.9366$ & $1.7826+/-1.7703$ & 0.59 \\
\hline Rockstar & Pre Edrink & Post Edrink & Pvalue \\
\hline EO & $0.6439+/-0.5295$ & $0.4873+/-0.3166$ & 0.80 \\
\hline $\mathrm{EC}$ & $1.2861+/-0.5582$ & $1.0481+/-0.6149$ & 0.77 \\
\hline EOHUD & $1.1330+/-0.7968$ & $0.9429+/-0.4550$ & 0.67 \\
\hline ECHUD & $2.4397+/-1.5348$ & $1.4300+/-1.1409$ & 0.23 \\
\hline
\end{tabular}

$\mathrm{EO}=$ Eyes Open, $\mathrm{EC}=$ Eyes Closed, $\mathrm{HUD}=\mathrm{Head}$ up and down, Motor = holding a cup filled with water Table 4: MEDIO-LATERAL Jerk during balance tasks. Results of repeated measure ANOVA between preenergy drink and post-energy drink. Significance level set at $\mathrm{p} \leq 0.05$.

Table 5 shows the total sway outcomes during the different balance tests for all participants pre and post-energy drinks.

\begin{tabular}{|c|c|c|c|}
\hline \multicolumn{4}{|c|}{ SWAY $\left(\mathbf{m}^{2} / \mathbf{s}^{4}\right)$} \\
\hline Redbull & Pre Edrink & Post Edrink & $P$ value \\
\hline EO & $0.076+/-0.10$ & $1.38+/-3.26$ & 0.81 \\
\hline $\mathrm{EC}$ & $0.07+/-0.05$ & $0.07+/-0.04$ & 0.87 \\
\hline EOHUD & $0.258+/-0.49$ & $0.094+/-0.06$ & 0.17 \\
\hline ECHUD & $0.277+/-0.37$ & $0.192+/-0.18$ & 0.43 \\
\hline Bang & Pre Edrink & Post Edrink & Pvalue \\
\hline EO & $0.049+/-0.02$ & $0.025+/-0.02$ & 0.97 \\
\hline $\mathrm{EC}$ & $0.07+/-0.02$ & $0.085+/-0.08$ & 0.57 \\
\hline EOHUD & $0.06+/-0.03$ & $0.058+/-0.03$ & 0.97 \\
\hline ECHUD & $0.116+/-0.04$ & $0.125+/-0.09$ & 0.93 \\
\hline Rockstar & Pre Edrink & Post Edrink & Pvalue \\
\hline EO & $0.047+/-0.04$ & $0.041+/-0.02$ & 0.99 \\
\hline $\mathrm{EC}$ & $0.09+/-0.04$ & $0.06+/-0.01$ & 0.25 \\
\hline EOHUD & $0.127+/-0.12$ & $0.10+/-0.06$ & 0.81 \\
\hline ECHUD & $0.20+/-0.19$ & $0.119+/-0.06$ & 0.40 \\
\hline
\end{tabular}

Table 5: Sway during balance tasks. Results of repeated measure ANOVA between pre-energy drink and postenergy drink. Significance level set at $\mathrm{p} \leq 0.05$. 
Table 6 shows the neuromuscular activity data for the hand grip flexor musculature. Handgrip data were similar among the different groups; however, post-e drink data showed a decreased tendency in various timing variables. This reduction in time was more evident in the RedBull group during the EMG activity decay. This suggests that the RedBull participants showed an increased tendency to muscle fatigue compared to the other groups due to the quicker activity decay.

\begin{tabular}{|l|l|l|l|}
\hline Hand Grip EMG data & Pre Edrink & Post Edrink & P value \\
\hline Redbull & $8.9500+/-2.4933$ & $8.2167+/-1.5182$ & 0.92 \\
\hline onset & $5.1161+/-2.3070$ & $7.4522+/-2.2880$ & 0.75 \\
\hline Time to peak & $10.8072+/-0.3216$ & $11.1094+/-0.8023$ & 0.75 \\
\hline duration & $5.6911+/-2.4157$ & $3.6572+/-2.2154$ & 0.11 \\
\hline decay & Pre Edrink & Post Edrink & Pvalue \\
\hline Bang & $8.0562+/-0.5726$ & $7.6705+/-0.7733$ & 0.75 \\
\hline onset & $6.3990+/-1.9303$ & $6.7348+/-1.6338$ & 0.77 \\
\hline Time to peak & $10.8110+/-0.4443$ & $10.9719+/-0.6383$ & 0.85 \\
\hline duration & $4.4119+/-1.8224$ & $4.2371+/-1.7769$ & 0.81 \\
\hline decay & Pre Edrink & Post Edrink & P value \\
\hline Rockstar & $9.2024+/-2.6852$ & $9.5833+/-3.1942$ & 0.74 \\
\hline onset & $5.5486+/-2.7338$ & $6.0262+/-2.1835$ & 0.69 \\
\hline Time to peak & $11.1329+/-0.8555$ & $10.1357+/-3.5493$ & 0.25 \\
\hline duration & $5.5843+/-2.6448$ & $4.1095+/-2.0909$ & 0.21 \\
\hline decay & & \\
\hline All variables units are in seconds. & & \\
\hline
\end{tabular}

Table 6: EMG data for Hand Grip flexor muscles. Results of repeated measure ANOVA between pre-energy drink and post-energy drink. Significance level set at $\mathrm{p} \leq 0.05$.

\section{Discussion}

There were several aspects of energy drinks and performance that this study aimed to investigate. This study intended to determine the impact of EDs on balance, muscle activation/ fatigue as well and cognitive performance (specifically attention and memory) during multitasking activities. In the current study, the authors strived to determine the effect of caffeine and other energy drink ingredients on balance and lower extremity muscle activation. Further, this investigation endeavored to address a gap in the literature regarding the potentially detrimental effects of EDs on learning and health in students.

This research presented similarities between pre and post-energy drink consumption in all groups, and there were some groups' tendencies after energy drink consumption. Most notably, there was a propensity in the Redbull group to reduce jerk in the AP direction post-energy drink. Although this change was not significant, this finding shows reduced AP sway in this group following energy drink consumption, showing a slight improvement in stability and balance. One cause for the reduced sway in the Red Bull group could be associated with the amount of caffeine compared to bang and rockstar drinks. Red Bull had the lowest caffeine of the three beverages compared in this research $(111 \mathrm{mg}$ in a can of Red Bull vs. $160 \mathrm{mg}$ and $300 \mathrm{mg}$ in Rockstar and Bang). Rosario et al. [21] discovered increasing sway after caffeine consumption in similar age participants to this investigation. We relate the main difference and possible explanation for the reduced or improved sway to the amount of caffeine. Rosario et al. 's [21] research used $200 \mathrm{mg}$ of caffeine pills than $111 \mathrm{mg}$ that Red Bull contains.

The second outcome of the current study showed that the Red Bull group exhibited a shorter time to EMG decay in handgrip strength. This discovery indicates that this group was quicker to fatigue than the other following energy drink consumption. This finding is contrary to the limited literature on this subject, which may indicate that energy utilization can increase maximal voluntary isometric contraction [11], and a study that found a link between caffeine and increased repetition and power output during exercise [12]. Other studies have implied that EDs may lead to decreased blood flow to peripheral muscles, precipitating a surge in fatigue [14]. Blood flow reduction may explain the faster onset of fatigue time in the Red Bull participants found in this study. Interestingly, and as previously mentioned, Red Bull had the lowest amount of caffeine in the three drinks studied in this work. Based on this, it may be possible that certain caffeine levels may enhance performance after a specific threshold not reached by the caffeine content in Red Bull.

Finally, the third finding from this investigation showed no difference between pre and post-energy drink consumption in terms of cognitive and memory tasks. This discovery suggests that EDs had no impact, beneficial or detrimental to cognitive performance. Our findings correlate with some of the literature data, including a study done by Trunzo et al. in 2014 [10], which found that energy drink consumption decreased academic performance in college students. Further, our results also aligned with those of Tallis $\mathrm{J}$ et al. [22], where caffeine proved to be inefficient, enhancing balance and gait during cognitive dual tasks in an older group.

One limitation of this part of the study might be associated with the tool selected to measure memory performance. While this may be useful in populations with dementia and other diagnosed memory deficits, this tool may not have been the most appropriate for use in young, healthy adults. Future studies should investigate different memory methods that can be used as a baseline for young, healthy populations.

\section{Conclusion}

The ongoing study revealed several tendencies associated with Red Bull and its ingredients, such as the amount of caffeine in this particular beverage. It seems that a lower quantity of caffeine intake could improve balance in young, healthy adults. Furthermore, muscle fatigue was part of the trends established in this work during a handgrip test after EDC. 
In synopsis, this inquiry provides future directions for considering the effects of EDs on distinctive facets of performance in young adults. This investigation showed interesting discoveries that contrast with many claims generated by energy drink manufacturers, such as enhancing performance and mental focus. Additionally, this study's conclusions indicate that individual EDs may have some beneficial effects on muscle performance.

The primary constraint of this investigation was the limited sample size. It was problematic to enlist participants to consume EDs after not consuming caffeine for a minimum of 12 hours. Several volunteers were disqualified and had to reschedule because of caffeine consumption within 24 hours of testing. We were limited in our ability to continue recruiting participants due to COVID-19 examined only static balance, which is a task that is not inherently challenging for most healthy young adults.

Future investigations in this area may look at the effects of energy drink consumption on dynamic balance activities such as gait and other functional activities. Other cognitive performance aspects, such as reaction time and concentration, may be more pertinent to inspect as maximal voluntary isometric contraction, should be examined, as some studies have shown performance changes following energy drink consumption. Additionally, similar to Rosario et al.'s [23] neuromuscular protocol, we recommend seeking the effects of these three different drinks on neuromuscular timing for a comprehensive view of this beverage's impact, nevertheless, in a dynamic setting. This perspective is also applicable to athletes who routinely use EDs before workouts or competitions. It is likewise fundamental to delve into the effects of EDs in younger populations, such as those 14-18 years old, who are frequently the consumers of these EDs. One last note, as suggested by Harty, PS et al. [24], additional considerations could focus on data collection after one hour of caffeine/ED consumption.

Acknowledgements: Special thanks to Leslie Hadley and Dr. Maryvi Gonzalez-Sola for their assistant during this work Declaration

Funding: Supported by Texas Woman's University Small Grant Program

Conflicts of interest/Competing interests: Authors report no conflict or competing interest.

\section{Ethics approval: IRB approval TWU protocol \# 20107 \\ References}

1. Burrows, T., Pursey, K., Neve, M., \& Stanwell, P. (2013, March 1). What are the health implications associated with the consumption of energy drinks? A systematic review. Retrieved from https://academic.oup.com/nutritionreviews/ article/71/3/135/1898088.

2. De Sanctis, V., Soliman, N., Soliman, A. T., Elsedfy, H., Di Maio, S., El Kholy, M., \& Fiscina, B. (2017, August 23). Caffeinated energy drink consumption among adolescents and potential health consequences associated with their use: a significant public health hazard. Retrieved from https://www. ncbi.nlm.nih.gov/pubmed/28845841.

3. Wassef, B., Kohansieh, M., \& Makaryus, A. N. (2017, November 26). Effects of energy drinks on the cardiovascular system. Retrieved from https://www.ncbi.nlm.nih.gov/pmc/ articles/PMC5714807/.

4. Curran, C. P., \& Marczinski, C. A. (2017, December 1). Taurine, caffeine, and energy drinks: Reviewing the risks to the adolescent brain. Retrieved from https://www.ncbi.nlm.nih. gov/pubmed/29251842. restrictions. The other primary restraint was that this research in a younger population. Other parts of muscle performance, such

5. Fletcher, E. A., Lacey, C. S., Aaron, M., Kolasa, M., Occiano, A., \& Shah, S. A. (2017, April 26). Randomized Controlled Trial of High-Volume Energy Drink Versus Caffeine Consumption on ECG and Hemodynamic Parameters. Retrieved from https:// www.ncbi.nlm.nih.gov/pubmed/28446495.

6. Lippi, G., Cervellin, G., \& Sanchis-Gomar, F. (2015). Energy Drinks and Myocardial Ischemia: A Review of Case Reports. Cardiovascular Toxicology, 16(3), 207-212. doi: 10.1007/ s12012-015-9339-6

7. Svatikova, A., Covassin, N., Somers, K. R., Somers, V., Soucek, F., Kara, T., \& Bukartyk, J. (2015, January 1). A randomized trial of cardiovascular responses to energy drink consumption in healthy adults. Retrieved from https://mayoclinic.pure.elsevier. $\mathrm{com} / \mathrm{en} /$ publications/a-randomized-trial-of-cardiovascularresponses-to-energy-drink-co.

8. Sanchis-Gomar, F., Leischik, R., \& Lippi, G. (2016). Energy drinks: Increasing evidence of negative cardiovascular effects. International Journal of Cardiology, 206, 153. doi: 10.1016/j. ijcard.2016.01.107

9. Breda, J. O. J., Whiting, S. H., Encarnaã§̃̃£O, R., Norberg, S., Jones, R., Reinap, M., \& Jewell, J. (2014). Energy Drink Consumption in Europe: A Review of the Risks, Adverse Health Effects, and Policy Options to Respond. Frontiers in Public Health, 2. doi: 10.3389/fpubh.2014.00134

10. Trunzo, J. J., Samter, W., Morse, C., McClure, K., Kohn, C., Volkman, J. E., \& O'Brien, K. (2014). College Students' Use of Energy Drinks, Social Problem-Solving, and Academic Performance. Journal of Psychoactive Drugs, 46(5), 396-401.

11. Collier, N. B., Hardy, M. A., Millard-Stafford, M. L., \& Warren, G. L. (2016). Small Beneficial Effect of Caffeinated Energy Drink Ingestion on Strength. Journal of Strength and Conditioning Research, 30(7), 1862-1870. doi: 10.1519/ jsc.0000000000001289

12. Mccormack, W. P., \& Hoffman, J. R. (2012). Caffeine, Energy Drinks, and Strength-Power Performance. Strength and Conditioning Journal, 34(4), 11-16. doi: 10.1519/ ssc.0b013e $31825 \mathrm{f} 4 \mathrm{~d} 7 \mathrm{e}$

13. Dawes, J., Ocker, L. B., Temple, D. R., Spaniol, F., Murray, A. M., \& Bonnette, R. (2011). Effect of a pre-exercise energy drink (Redline $\left.{ }^{\circledR}\right)$ on upper-body muscular endurance performance. Journal of the International Society of Sports Nutrition, $8(\mathrm{~S} 1)$. doi: 10.1186/1550-2783-8-s1-p18

14. Higgins, J.P., Babu, K., Deuster, P.A., \& Shearer, J. (2018). Energy Drinks: A Contemporary Issues Paper. Curr Sports Med Rep. 17(2):65-72. doi: 10.1249/JSR.0000000000000454.

15. Nowak, D., Gośliński, M., \& Nowatkowska, K. (2018, March 19). The Effect of Acute Consumption of Energy Drinks on Blood Pressure, Heart Rate and Blood Glucose in the Group of Young Adults. Retrieved from https://www.ncbi.nlm.nih.gov/ pubmed/29562659

16. McDonald, J.S., McDonald, R.J., Comin, J., Williamson, E.E., Katzberg, R.W., Murad, M.H., Kallmes, D.F. (2013). Radiology. 267(1):119-28. doi: 10.1148/radiol.12121460. Epub 2013 Jan 14.

17. Moustaka, V., Vakali,A., G. Anthopoulos,L.,(2018). A Systematic Review for Smart City Data Analytics. ACM Computing Surveys 51(5):1-41. DOI:10.1145/3239566

18. Trevino, M. A., Coburn, J. W., Brown, L. E., Judelson, D. A., \& Malek, M. H. (2015). Acute Effects of Caffeine on Strength and Muscle Activation of the Elbow Flexors. Journal of Strength and Conditioning Research, 29(2), 513-520. doi: 10.1519/ jsc.0000000000000625

19. Higgins, J. P., Yang, B., Herrin, N. E., Yarlagadda, S., Le, G. T., Ortiz, B. L., ... Infanger, S. C. (2017). Consumption of energy beverage is associated with attenuation of arterial endothelial flow-mediated dilatation. World Journal of Cardiology, 9(2), 162. doi: $10.4330 /$ wjc.v9.i2.162 
20. Shumway-Cook, A., Woollacott, M.H. (2017). Changes in posture control across the life span--a systems approach. Phys Ther, 70(12):799-807. doi: 10.1093/ptj/70.12.799.

21. Rosario, M. G., Collazo, H., Mateo, M., Gonzalez-Solo, M., \& Bayron, F. (2017). Increased Static Postural Sway After Energy Drink Consumption a Randomized Trial. F1000 Research., doi: 10.12688/f1000research.12565.1

22. Tallis, J; Bradford, C; Duncan, MJ; Leddington-Wright, S; Higgins, MF; Hill, M. The Effect of Acute Caffeine Ingestion on Cognitive Dual-Task Performance during Assessment of Static and Dynamic Balance in Older Adults. Nutrients, 2020 vol. 12(12)
23. Rosario, M.G., Jamison, L., \& Hyder, A. (2020). The Influence of Energy Drinks on Lower Limb Neuromuscular Timing and Postural Sway in Healthy Young Adults. J Pub Health Issue Pract 4(1):163. https://doi.org/10.33790/jphip1100163

24. Harty, PS; Zabriskie, HA; Stecker, RA; Currier, BS; Tinsley, GM; Surowiec, K; Jagim, AR; Richmond, SR; Kerksick, CM. Caffeine Timing Improves Lower-Body Muscular Performance: A Randomized Trial. Front Nutr, 2020 vol. 7 p. 585900 